\title{
Single-Photon Two-Qubit Logic Gates
}

\author{
Marco Fiorentino*, Taehyun Kim* and Franco N. C. Wong* \\ ${ }^{*}$ Research Laboratory of Electronics, Massachusetts Institute of Technology, Cambridge, MA 02139
}

\begin{abstract}
We present experimental results of deterministic linear optical Controlled-NOT and SWAP gates for single-photon two-qubit quantum logic. In our implementation one qubit is encoded in the polarization degree of freedom of the photon and the other is encoded in the momentum. Momentum- and polarization-controlled NOT gates are demonstrated. They are then combined to form a SWAP gate to convert pairs of momentum-entangled photons into polarization-entangled photons.
\end{abstract}

Linear optical quantum computation (LOQC) has recently attracted great interests following the suggestion [1] that a scalable quantum computer based on linear optical components is possible. It has also been known that linear optical systems could achieve non-scalable quantum computation by encoding multiple qubits in several degrees of freedom of a single photon [2]. Experiments in the latter were limited to a few qubits due to the complexity of the optical setup [3] and they did not use entanglement resources. Recently, however, several groups have proposed the use of deterministic logic gates in conjunction with sources of entangled or hyperentangled (i.e., entangled in more than one degree of freedom) photons to execute simple quantum protocols. The combination of deterministic logic and entangled photons can be used for one-shot demonstration of nonlocality with two observers [4], complete analysis of Bell's states [5], cryptographic protocols [6], and quantum games [7]. These proposed experiments rely on the ability to create hyperentangled states and successively project them onto suitable sets of basis states for measurement. Hyperentangled photons can be readily used as quantum resources if their entanglement can be routinely manipulated by efficient deterministic one- and two-qubit gates. The utility of single-photon two-qubit (SPTQ) quantum information processors depends on one's ability to create and manipulate the individual qubits (qubit rotation) and to implement two-qubit quantum gates in a robust experimental setup. We here present an implementation of SPTQ logic based on the encoding of qubits in the momentum and polarization degrees of freedom of single photons. The interest of such implementation resides in the ease of implementing one- and two-qubit gates and the ability to generate photons pairs that are entangled in one or both degrees of freedom. In this contribution we present the implementation of two-qubit gates for this logic protocol and their application of logic gates to entanglement manipulation.

Our SPTQ logic protocol encodes two qubits in a single photon: the first qubit is represented by the photon polarization and the second by the position of the photon within the beam. The combination of the two possible polarization states ( $H$ and $V$ for horizontally and vertically polarized photons) and the two momentum states ( $T$ and $B$ for photons in the top and bottom part of the beam) constitute the four states of the two-qubit computational basis. To manipulate this system, in addition to one-qubit gates (that can be implemented with wave plates and beam splitters) one has to build two types of CNOT gates. In the first type the momentum is the control qubit while the polarization is the target (M-CNOT). In the second type the roles are reversed with the polarization being the control qubit and the momentum the target (P-CNOT). The implementation of these two types of gates is relatively straightforward [8]. The M-CNOT gate can be realized with a half-wave plate (HWP) oriented at $45^{\circ}$ relative to the horizontal position and inserted in the path of the $B$ beam. The P-CNOT gate is a polarization Sagnac interferometer containing a dove prism whose base is oriented at a $45^{\circ}$ angle relative to the horizontal plane. The input polarizing beam splitter directs horizontally (vertically) polarized input light to travel in a clockwise (counterclockwise) direction. As viewed by each beam, the dove prism orientation is different for the two counter-propagating beams such that the transformation of the input spatial image differs for the horizontal $(H)$ and vertical $(V)$ polarizations. Specifically, the top-bottom $(T-B)$ sections of the input beam are mapped onto the right-left $(R-L)$ sections of the output beam for $H$-polarized light but onto the $L-R$ sections for $V$-polarized light. If we identify $|H\rangle,|T\rangle$, and $|R\rangle$ with the logical $|0\rangle$ and $|V\rangle,|B\rangle$, and $|L\rangle$ with the logical $|1\rangle$, this setup implements a P-CNOT gate. We note that both implementations have been shown to be highly stable and do not require active path-length stabilization, which is a distinct advantage over similar proposed

CP734, Quantum Communication, Measurement and Computing, edited by S. M. Barnett et al.

(C) 2004 American Institute of Physics 0-7354-0216-7/04/\$22.00 


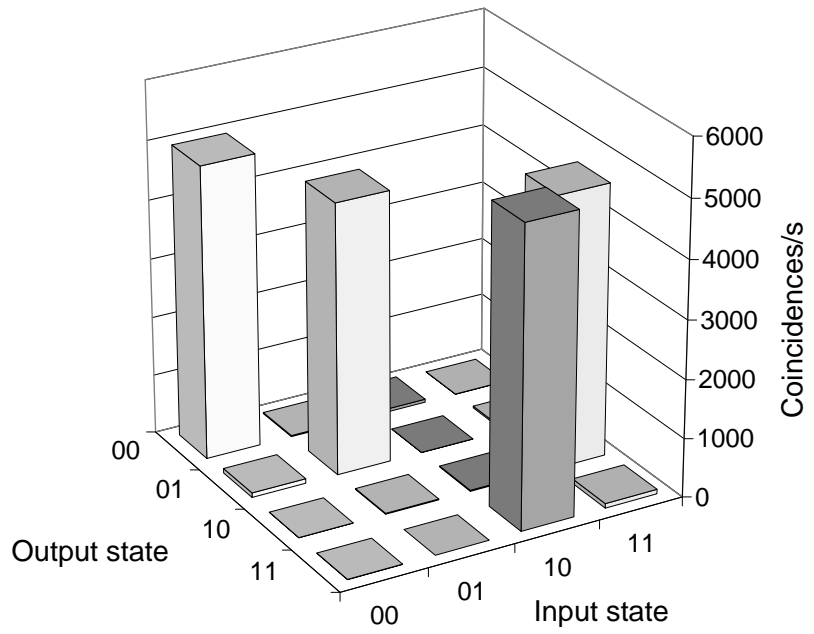

FIGURE 1. Coincidence count rates as a function of the projected output state for a given input state.

protocols $[4,5]$. Figure 1 shows the truth table of the P-CNOT gate that agrees very well with the ideal table for a CNOT gate. We have used [8] the P-CNOT gate to entangle momentum and polarization of a single photon when the input photon is in the state

$$
|\Psi\rangle_{1}=\frac{1}{\sqrt{2}}(|H\rangle+|V\rangle) \otimes|T\rangle
$$

A combination of CNOT gates can be used to build a SWAP gate as described, for example, in Ref. [9]. A SWAP gate exchanges the values of two arbitrary qubits. For example, when applied to the arbitrary two-qubit product state $(\alpha|T\rangle+\beta|B\rangle) \otimes(\gamma|H\rangle+\delta|V\rangle)$, a SWAP gate transforms it into the state $(\gamma|T\rangle+\delta|B\rangle) \otimes(\alpha|H\rangle+\beta|V\rangle)$. When applied on a qubit that is part of an entangled pair of qubits the SWAP gate transfers the entanglement to the other qubit, which may be more conveniently manipulated. In our experiment we used down-converted photon pairs from a periodically poled potassium titanyl phosphate (PPKTP) crystal [10]. These photons show momentum entanglement similar to the type demonstrated by Rarity and Tapster in Ref. [11]. In our notation the state of the photons at the output of the crystal is

$$
|\Psi\rangle_{2}=\frac{1}{\sqrt{2}}\left(\left|T_{S} B_{I}\right\rangle+\left|B_{S} T_{I}\right\rangle\right) \otimes\left|H_{S} V_{I}\right\rangle
$$

where the subscripts $S$ and $I$ refer to the signal and idler photons, respectively. A SWAP gate can be obtained with a sequence of a M-CNOT followed by a P-CNOT and a M-CNOT. Applying a SWAP gate to each of the signal and idler photons allows us to exchange the qubits of both photons and we obtain the polarization-entangled state

$$
|\Psi\rangle_{3}=\frac{1}{\sqrt{2}}\left|L_{S} R_{I}\right\rangle \otimes\left(\left|H_{S} V_{I}\right\rangle+\left|V_{S} H_{I}\right\rangle\right)
$$

where $L$ and $R$ are the states of the computational basis for the momentum qubit after the P-CNOT. Figure 2 shows the experimental setup used to verify entanglement swapping. We used pairs of down-converted photons from a 1-cm-long PPKTP crystal that was continuous-wave pumped at $398.5 \mathrm{~nm}$ for type-II phase-matched collinear frequency-degenerate parametric down-conversion [12]. The momentum modes were chosen with two apertures after the gates and the same physical gates were used to manipulate both photons of the pair. After the polarization Sagnac interferometer that implements the P-CNOT gate the state of the photon pair is

$$
\left|\Psi^{\prime}\right\rangle_{3}=\frac{1}{\sqrt{2}}\left|L_{S} R_{I}\right\rangle \otimes\left(\left|H_{S} H_{I}\right\rangle+\left|V_{S} V_{I}\right\rangle\right)
$$




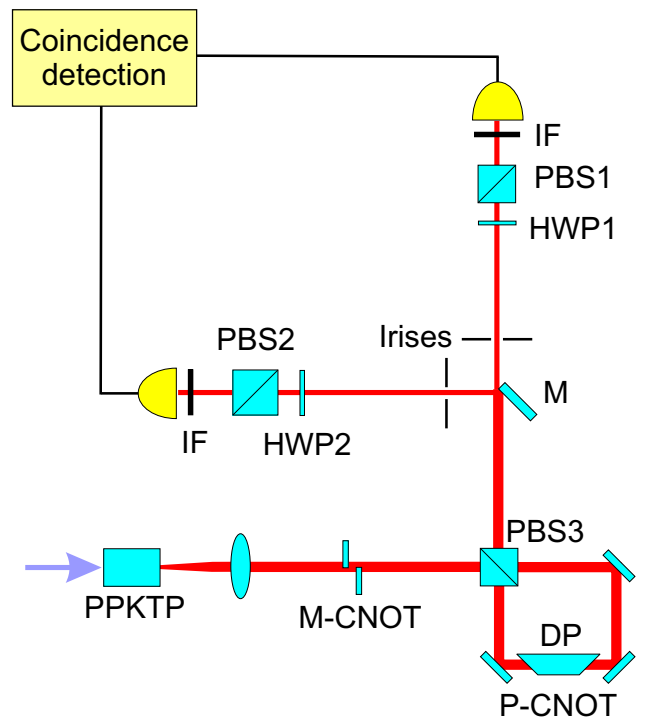

FIGURE 2. Schematic of the entanglement swapping experimental setup. PPKTP: periodically poled KTP crystal. PBS: polarizing beam splitter. DP: dove prism. HWP: half-wave plate. IF: 1-nm interference filter. M: mirror. P-CNOT: polarization-controlled NOT gate. M-CNOT: momentum controlled NOT gate.

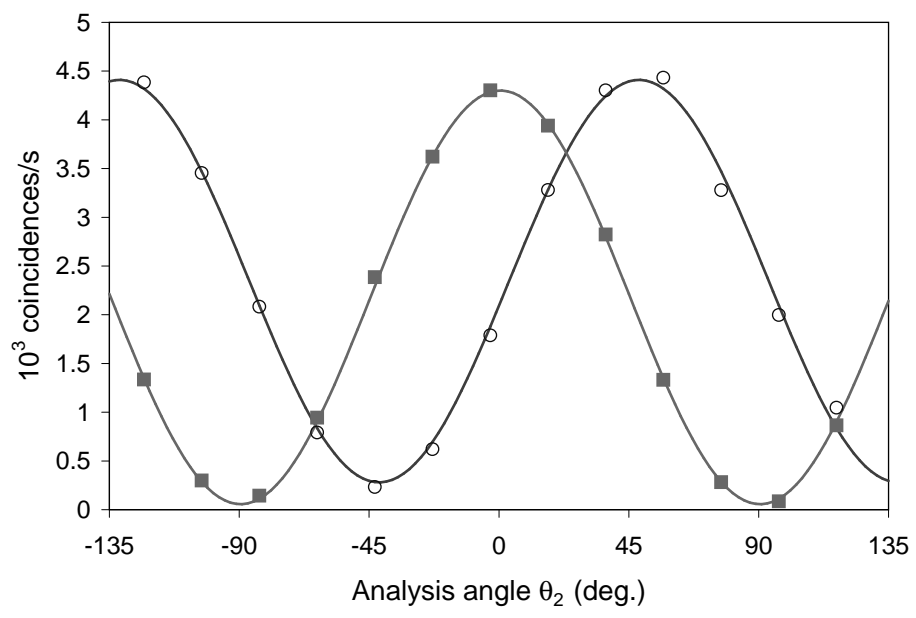

FIGURE 3. Coincidence rates as a function of the polarization analysis angle $\theta_{2}$ in arm 2 when the analyzer in arm 1 was set at an angle $\theta_{1}=0^{\circ}$ (solid squares) and $45^{\circ}$ (open circles). The lines are sinusoidal fits to the data.

Observe that this state is polarization entangled. We separated signal and idler photons using the mirror M shown in Fig. 2 that reflected only the right section of the beam. Signal and idler beams were then separately sent through a 2.2$\mathrm{mm}$ iris, a polarization analyzer formed by a half-wave plate and a polarizer, and a 1-nm interference filter centered at $797 \mathrm{~nm}$. Besides being used for polarization analysis, half-wave plate HWP2 in Fig. 2 assumed the role of the second M-CNOT gate, thus completing the SWAP circuit. Figure 3 shows the typical coincidence fringes for the resultant polarization-entangled photons, thus demonstrating that the swapping of entanglement has been achieved. When the analyzer in arm 1 was set at $0^{\circ}$ (solid squares) a visibility $V_{0}=97 \pm 2 \%$ was observed; at $45^{\circ}$ (open circles) we obtained a visibility $V_{45}=88 \pm 2 \%$.

In conclusion we have demonstrated a set of quantum gates for single-photon two-qubit logic. These constitute an essential set of gates in the SPTQ quantum logic family comprising linear optical P-CNOT and M-CNOT gates that are robust and do not need active length stabilization. In addition we have combined C-NOT gates to form a 
SWAP gate which we applied to momentum-entangled photons to transfer the entanglement from the momentum to the polarization degree of freedom. Our experiments open the way to the demonstration of more complex SPTQ manipulation of entanglement including the manipulation of 3- and 4-photon states. This type of few-qubit quantum information processing is at the core of a number of applications ranging from single-shot two-observers demonstration of nonlocality [4] to two-qubit quantum key distribution [6].

\section{ACKNOWLEDGMENTS}

This work was supported by the the DoD Multidisciplinary University Research Initiative (MURI) program administered by the Army Research Office under Grant DAAD-19-00-1-0177.

\section{REFERENCES}

1. E. Knill, R. Laflamme, and G. J. Milburn, Nature 409, 46 (2001).

2. N. J. Cerf, C. Adami, and P. G. Kwiat,Phys. Rev. A 57, R1477 (1998); J. C. Howell and J. A. Yeazell, Phys. Rev. A 61, 052303 (2000); B. G. Englert, C. Kurtsiefer, and H. Weinfurter, Phys. Rev. A 63, 032303 (2001).

3. P. G. Kwiat, J. R. Mitchell, P. D. D. Schwindt, and A. G. White, J. Mod. Opt. 47, 257 (2000); Y. Mitsumori, J. A. Vaccaro, S. M. Barnett, E. Andersson, A. Hasegawa, M. Takeoka, and M. Sasaki, Phys. Rev. Lett. 91, 217902 (2003).

4. Z.-B. Chen, J.-W. Pan, Y.-D. Zhang, C. Brukner, and A. Zeilinger, Phys. Rev. Lett. 90, 160408 (2003).

5. S. P. Walborn, S. Pádua, and C. H. Monken, Phys. Rev. A 68, 042313 (2003).

6. M. Genovese and C. Novero, Eur. Phys. J. D 21, 109 (2002).

7. K.-Y. Chen, T. Hogg, and R. Beausoleil, Quant. Info. Proc. 1, 449 (2002).

8. M. Fiorentino and F. N. C. Wong, Phys. Rev. Lett. 93, 070502 (2004).

9. M. A. Nielsen and I. L. Chuang, Quantum Computation and Quantum Information (Cambridge University, Cambridge, 2000) p. 23.

10. M. Fiorentino, T. Kim, and F. N. C Wong, quant-ph/0407136.

11. J. G. Rarity and P. R. Tapster, Phys. Rev. Lett. 64, 2495 (1990).

12. C. E. Kuklewicz, M. Fiorentino, G. Messin, F. N. C. Wong, and J. H. Shapiro, Phys. Rev. A 69, 013807 (2004); M. Fiorentino, G. Messin, C. E. Kuklewicz, F. N. C. Wong, and J. H. Shapiro, Phys. Rev. A 69, 041801(R) (2004). 